sind wie für $m>n_{0}$. Hierzu tritt nach meiner Theorie noch die Einschränkung, daß für $m=n_{0}$ die $\log A_{m}$ und ihre ersten Differentialquotienten stetig verlaufen müssen, $d . h$. die beiderseitigen Interpolationsformeln dieselben Werte ergeben müssen. Als Interpolationsformel mag eine quadratische Form genügen, wenn man sich nicht zu weit von $m=n_{0}$ entfernt, indessen dürfen die Grenzen des Intervalles nicht zu eng. genommen werden, weil sonst die Bestimmung der Unbekannten selbstverständlich an Sicherheit einbüßt.

Danach habe ich die $\log A_{m}$ im Intervall $m=4$ bis $m={ }_{1} 4$ durch die Formeln einheitlich ausgeglichen

$$
\begin{array}{ll}
\text { für } m<n_{0} & \log A_{m}=\alpha+\beta\left(m-n_{0}\right)-\gamma\left(m-n_{0}\right)^{2} \\
\text { für } m>n_{0} & \log A_{m}=\alpha+\beta\left(m-n_{0}\right)-\gamma_{1}\left(m-n_{0}\right)^{2} .
\end{array}
$$

Es stehen dann 11 Gleichungen mit den 5 Unbekannten $\alpha$, $\beta, \gamma, \gamma_{1}$ und $n_{0}$ zur Verfügung. Wenn man über Näherungswerte der Unbekannten verfügt, sind ihre Verbesserungen durch die Behandlung nach der M. d. kl. Qu. zu gewinnen. Die Bedingungsgleichungen sind

$$
\begin{gathered}
A=\left(\mathrm{d} \alpha-\beta \mathrm{d} n_{0}\right)+\left(m-n_{0}\right) \mathrm{d} \beta+\mathrm{d} n \cdot 2 \gamma\left(m-n_{0}\right) \\
-\mathrm{d} \gamma\left(m-n_{0}\right)^{2} \quad m<n_{0} \\
\text { bezw. } A=\left(\begin{array}{c}
\left.\mathrm{d} \alpha-\beta \mathrm{d} n_{0}\right)+\left(m-n_{0}\right) \mathrm{d} \beta+\mathrm{d} n \cdot 2 \gamma_{1}\left(m-n_{0}\right) \\
-\mathrm{d} \gamma_{1}\left(m-n_{0}\right)^{2} \quad m>n_{0} .
\end{array}\right.
\end{gathered}
$$

Die Rechnung wurde unter den bekannten VorsichtsmaBregeln ausgeführt, sodaß die Anführung des Resultats genügt. Da zuerst von etwas abweichenden Näherungswerten ausgegangen wurde, wurden 2 Näherungen ausgeführt, was jedenfalls genügt, da ja die größte Genauigkeit zu erhalten nicht beabsichtigt werden konnte. Es ergab sich:

$$
\text { für } m<n_{0} \quad \begin{aligned}
\log A_{m}=0.4595 & +0.46930(m-8.8803) \\
& -0.005739(m-8.8803)^{2} .
\end{aligned}
$$

Für $m>n_{0}$ bleiben selbstverständlich die beiden ersten Glieder ungeändert, das dritte Glied erhält aber den Koeffizienten -0.013590 . Die Darstellung der Tabellenwerte $B$ durch die Formel $F$ ist eine fast vollkommene:

München, I 92 I Mai.

$\begin{array}{rrrrrrrr}m & F & B & \Delta & m & F & b & \Delta \\ 4 & -1.967 & -1.967 & 0 & 10 & +0.968+0.967 & -1 \\ 5 & -1.448 & -1.450 & -2 & 11 & +1.393+1.393 & 0 \\ 6 & -0.940 & -0.942 & -2 & 12 & +1.792 & +1.791 & -1 \\ 7 & -0.443 & -0.443 & 0 & 13 & +2.162 & +2.161 & -1 \\ 8 & +0.042 & +0.044 & +2 & 14 & 12.505+2.505 & 0\end{array}$

Als $m$. F. ergaben sich: $\varepsilon_{\gamma}= \pm 0.00026 \quad \varepsilon_{\gamma_{1}}= \pm 0.00025 \quad \varepsilon_{n_{0}}= \pm 0.14$

Danach ist $n_{0}$ weniger sicher bestimmt als die andern Unbekannten. Der Betrag der Unstetigkeit im 2. Differentialquotienten ist $0.016 \pm 0.0007$, wodurch seine Existenz mit fast absoluter Sicherheit festgestellt ist. Wohl mit Bestimmtheit ist anzunehmen, daß die Tabellenwerte für $\log A_{m}$ ohne Rücksicht auf das Vorkommen einer Unstetigkeit oder einer schnell vor sich gehenden Änderung aufgestellt worden sind. Damit ist selbstverständlich die Tendenz zu einer größeren Ausgeglichenheit verbunden, welche die "Sprünge * verkleinern muß. Deshalb ist $z u$ vermuten, daß der gefundene Betrag 0.016 zu klein, vielleicht merklich zu klein sein dürfte. Deshalb war das a. a. $O$. von mir angewandte Veriahren, das ich, um unabhängig von der Störung erster Art $z \mathfrak{u}$ sein, anzuwenden gezwungen war und das darin bestand, daß ich die 2 . Differenzen, welche ganz in der Nähe der kritischen Größe $n_{0}$ vorher und nachher lagen, in 2 wei Mittel vereinigte und voneinander subtrahierte, ein durchaus zulässiger Notbehelf. Die Rücksichtnahme auf die angezeigte Unstetigkeit bei der Aufstellung der Tafelwerte wird immerhin einige Vorsicht und Schwierigkeit bereiten. Das ist aber wohl kein Grund für ihre Ignorierung. Das vorliegende Material gibt, wie gezeigt, die von meiner Theorie lange vorher verlangten Unebenheiten in deutlichster Weise. Dadurch ist das vielleicht wichtigste Resultat derselben, nämlich der bestimmbaren Endlichkeit des Systems, selbstverständlich in vernünftiger Ausdeutung, von neuem erwiesen. Es wäre doch ein höchst merkwürdiger Zufall, wenn die eińer Uṇstetigkeit ähnlichen Veränderungen im 2. Differentialquotienten, welche von einer Theorie vorausgesagt worden sind und die sich dazu in einem von unbeteiligter Seite herbeigeschafften Material ganz deutlich zeigen, nicht reell sein sollten.

\title{
H. Seeliger.
}

\section{Osservazioni a stima della Variabile S Sagittae. Di G. B. Lacchini.}

Dal 22 giugno al I5 dicembre I9I furono fatte 60 osservazioni a stima per mezzo di un binocolo galileano a 3 ingr.; le stelle di confronto sono le seguenti:

$\begin{array}{llll}A=\zeta \text { Sagittae } & 4^{\mathrm{m}} \cdot 95 & c=\times_{3} \text { Sagittae } & 5^{\mathrm{m}} 54 \\ B=\eta 》 & 5.26 & d=\mathrm{I}_{5} \gg & 5.90 \\ a=\mathrm{II}_{1} \gg & 5.36 & e=9 》 & 6.24 \\ b=14 & 5.47 & f=+16^{\circ} 4086 & 6.76 .\end{array}$

Le grandezze delle prime due stelle sono prese da H. A. Vol. 50, quelle delle altre dal Vol. 63 ; le differenze di grandezza fra l'una e l'altra furono spesso stimate diverse da quelle date, come è visibile dallè stime della Var.; ciò si deve forse al colore giallognolo di quasi tutte le stelle di confronto. In base agli elementi:

$$
\text { Mass. }=2409863 \cdot 324+8{ }_{3} 8 \times 16 \times 3 \cdot E
$$

le osservazioni si riferiscono alle epoche 1376 a 1397 , la curva media ottenuta è pertanto quella dell'epoca 1386 .

La rappresentazione grafica della curva di luce, ottenuta con la perequazione delle osservazioni a 5 a 5 (Tabella II), ci mostra come questa Var. difficilmente possa essere messa fra quelle del tipo $\delta$ Cephei: mostra invece qualche rassomiglianza con quelle del tipo $\beta$ Lyrae, quantunque piuttosto che un vero minimo secondario fra due massimi abbia luogo un massimo a 2.45 dopo il minimo alla gr. 5.30, poi evvi una pausa di $0^{d} 70$, quindi un altro massimo dopo ${ }^{d}{ }^{d} 8$ alla

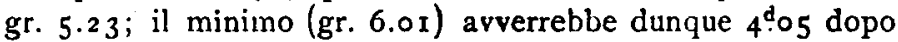
il massimo principale ed avremo $M-m=4.33$.

Riesce quindi difficile ricavare il residuo $(\mathrm{O}-\mathrm{C})$ della data del massimo, mentre il minimo è ben determinato; esso 
ha luogo a $6^{\text {do }}$. 5 dopo la data del mass. calcolato; ammettendo come valore $(M-m)$ 2 60 (Hartwig), avremo per il minimo un residuo $(\mathrm{O}-\mathrm{C})=+\mathrm{od}^{\mathrm{d}} \mathrm{2}$; ma quando si prenda invece come data del massimo il punto più alto della curva abbiamo per esso il residuo $(\mathrm{O}-\mathrm{C})=+2^{\text {d }}$. $\mathrm{a}$. Il minimo osservato (I386-I387) puó essere fissato alla data 2421486.289 . Tabella I.

1917 T.m. Gr. Fase Confronti

$I^{*}$ Giug. $22 \quad 8^{\mathrm{h}} 53^{\mathrm{m}} 5.9467=e, 4 f, d_{4}$

$2 *$ * $239406.9794 d 2, \mathrm{r} c$

$3 \gg 25 \quad 922 \quad 0.5852=b, 1 c, 2 d, a \mathrm{I}$

$4 \gg 26$ ז2 30 1.7 $157=B, 1 a, 2 b, 2 c, 5 d$

$5 \gg 2710 \circ 2.6116=a, B_{2},=b$, i $c$

$6 \gg 28$ I2 $35 \quad 3.7192 \quad a_{3}, b_{3},=c,=d, B_{4}$

$7 \gg 29 \quad 12 \quad 37 \quad 4.7206 \quad B_{4}, a 1, c 2, b 2,2 d$

$8^{*}$ Lugl. $4 \quad 933$ I.2 I12 $A$ I, $=B$, г $a$.

$9^{*} \gg 6 \quad 6 \quad 9 \quad 2 \quad 3.1897 \quad a_{2},=e, b 1,2 d$

I0* $\gg 79254.2057 a_{4}, b_{3}, c_{2}, d_{1}, 3 e$

$1^{*} \star 884 \mathrm{I} 5.175 \mathrm{I} a_{3}, b_{3}, c_{3}, d_{\mathrm{I}}, 2 e$

I $2 *$ I0 I0 $54 \quad 7.2675 \quad a 2, c 2,=b$, I $d, 5 e$

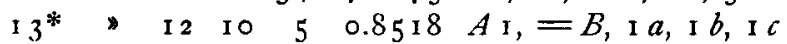

$14 \gg 13146 \quad 2.0192=B, 1 a, A 1,2 b, 2 c, 4 d$

$15 \gg$ I5 8 50 $3.7998 \quad 3 e, a_{5}, c_{3}, b_{3},=d$

$16 》 179205.7206 \quad a \circ b 2 c 3 d \circ S_{2}<4 f$

$17 》$ I 8 I3 $407.0011=b, a_{2}, 62,2 d$

I $8 \gg$ I9 I० I $2 \quad 7.8567 \quad a \mathrm{I},=c, \mathrm{I} b, 3 d$

г9 $920 \quad 9$ г 20.4334 I $a_{1}=B, A_{2}$

$20 \gg 2$ I I $3 \quad 52$ I.6279 I $a, 3 c, A_{3}, B_{3}$

2 I $\gg 22$ I0 $24 \quad 2.4834 \quad 2 a, B$ I, $A$ I

$22 》 239924 \quad 3.4418 \quad a 2, c 2,=b, 2 d$

$23 \gg 24$ I0 $94.4730 \quad a 3, b 2, c 2$, Id

$24 \gg 25950.5 .4598$ a $5, d_{3}, 2 c$

$25 \quad 27$ I $3 \quad 7 \quad 7.5966 \quad a 2, b \mathrm{I}, c \mathrm{I}, 2 d$

$26 *$ Agos. $4887.0074 a 5, b 5, c_{4}, 2 e, d 2$

$27 》 8928 \quad 2.68 \mathrm{I2}$ I $a, B \mathrm{I}, 2 b$, I $c$

$28 》 9 \quad 93^{2} \quad 3.6840 \quad a_{4}, b_{3}, c 2,=d, 3^{e}$

$29 \gg$ I $2 \quad 8206.6340 a 4, c 2, b 2, d 1,3 e$

30 I $4 \quad 8 \quad 7 \quad 0.2334$ J $a, B$ I, $A$ I

$3 \mathrm{I} \gg 23 \quad 8 \quad 49 \quad 0.8910$ I $a, 2 b, 2 c, B$ I

$32 \% \quad 28 \quad 7 \quad I_{7} 5.827 \mathrm{I} a_{5}, b_{4}, c_{3}, d_{\mathrm{I}, 3} e$

33 Sett. $4 \quad 8 \quad 33 \quad 4.4983 \quad a_{4}, b_{4}, c_{3}, d \mathrm{I}, 3 e$

$34 \gg \quad 5 \quad 8 \quad 5 \quad 5.4788 \quad a_{5}, d_{3}, 2 e$

$35 \quad 6 \quad 8456.5066 \quad a_{4}, b_{3}, c_{2},=d, 3 e$

36 " $9 \quad 7 \quad 35 \quad 1.0764=a, B_{1}, A 1,=b$

37 I0 8 10 $2.1007 \quad a_{\mathrm{I}}=B, A$ I

$38 \gg$ I $7243.0687=a,=b, 2 c, B 2, A \dot{3}$

39

40
724

744

$\begin{array}{lll}7 & 47\end{array}$
$6.0826 d_{3},=e, 3 f$

8.0847 I $a, 2 b, 4 c,=B, A_{2}$

\subsection{2}

5.33

$5 \cdot$ I 9

5.49

5.72

6.03

5.62

5.98

5.33

5.82

5.82

$5 \cdot 32$

$5 \cdot 31$

5.90

5.88

6.03

5.82

$5 \cdot 3 \mathrm{I}$

5. 19

5.37

6.30

5.22

Faenza, 1921 Febbraio Io.

1917 T.m. Gr. Fase

Confronti

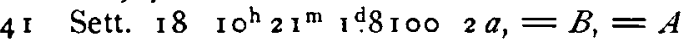

$42 》 20 \quad 733.6726=a$, I $b, 3 c, B_{2}$

$43 \gg 22 \quad 740 \quad 5.6982 \quad a_{5}, b_{4}, c_{3}, d_{2}, 2 e$

$44^{*} \gg 25 \quad 6 \quad 48 \quad 0.2805 \quad 3 a, 2 b, B$ I, $A$ I, $3 c$

$45 *$ Ott.

46

47

48

48 II

$\begin{array}{lll}7 & 4\end{array}$

$6.29 \mathrm{I} 6 a 6, b_{5}, c_{4}, d \mathrm{I}, 2 e$

$659 \quad 3.9065 \quad a_{3}, b_{3},=c,=d$

$739 \quad 5.9342 \quad a 5, b 4, c 2, d \mathrm{r}, 2 e$

$7507.9459 A \circ B$ I $S$ I $b$ I $a \circ c$

549 4.7 I.3 I $a_{5}, b_{4}, c_{3},=d, 2 e$

$\begin{array}{llll}5 & 35 & 5.7033 & a_{3}, b_{2},=c, 1 d\end{array}$

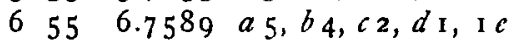

$53^{8} \quad 4.3^{2} 3^{8} \quad a .2, b \mathrm{r},=c, 2 d$

I.9585 $A 2, B 2,2 a, 2 b, 3 c$

$5.0096 a_{5}, b_{5}, c_{3}, d_{1}, 3 e$

$6.0075 a 5, b 3, c_{4}, d_{\mathrm{I}}, 2 e$

$7.9994 a_{1}, b_{1}=c, 2 d$

$2.24992 a$, I $b, 3 c,=B, A 2$

$7.1624 a_{3}, b_{2},=c_{12} d$

3.8258 a.2, $=b, 1,1,3 d$

$5.7807 a_{3}, b_{3}, c 1,=d, 3 e$
Mag.

$5^{\mathrm{m}} \mathrm{12}$

$5 \cdot 38$

5.94

5.20

5.98

5.72

5.90

5.26

5.90

5.67

5.92

5.59

5.26

5.88

5.92

$5 \cdot 57$

5.23

5.64

$5 \cdot 52$

5.78

Note 1, 2, 26, 57. Vento. - 9, 10, 26, 32, 33, 44, $45,55,56$. Luna forte. - 8. Eclissi totale di luna. - I I. Colore giallo arancio. - I 2, I 3 . Colore arancio giallo.

Tabella II.

\begin{tabular}{|c|c|c|c|c|c|}
\hline $\begin{array}{l}\text { Nr. } \\
30\end{array}$ & $\begin{array}{c}\text { Fase } \\
\text { Perequa }\end{array}$ & $\begin{array}{c}\text { Gr. } \\
\text { zione }\end{array}$ & $\begin{array}{l}\text { Nr. } \\
28\end{array}$ & $\begin{array}{c}\text { Fase } \\
3: 4 \text { I I }\end{array}$ & $\begin{array}{l}\text { Gr. } \\
5^{\mathrm{x}} \cdot 5.3\end{array}$ \\
\hline 44 & \multicolumn{2}{|c|}{$\begin{array}{c}\text { Perequazione } \\
5 \text { a } 5\end{array}$} & 6 & $3.54 \mathrm{I}$ & 5.60 \\
\hline I 9 & & & I 5 & 3.664 & 5.65 \\
\hline 3 & & & 59 & 3.734 & 5.66 \\
\hline I 3 & $0^{d} 477$ & $5^{\mathrm{m}} \cdot 3 \mathrm{I}$ & 46 & 3.787 & $5.7 \mathrm{I}$ \\
\hline 31 & 0.609 & $5 \cdot 3 I$ & 10 & 3.891 & 5.73 \\
\hline 36 & 0.767 & $5 \cdot 33$ & $5^{2}$ & 4.012 & $5.7 \mathrm{I}$ \\
\hline 8 & 0.923 & $5 \cdot 32$ & 23 & 4.147 & 5.68 \\
\hline 20 & I. I 3 I & 5.28 & 3.3 & 4.281 & 5.75 \\
\hline 4 & 1.304 & 5.29 & 49 & 4.443 & 5.79 \\
\hline $4 \mathrm{I}$ & 1.488 & 5.25 & 7 & 4.546 & $5 \cdot 75$ \\
\hline 53 & 1. 647 & 5.24 & 54 & 4.683 & $5.8 \mathrm{I}$ \\
\hline I 4 & 1.826 & 5.26 & I I & 4.823 & 5.83 \\
\hline 37 & 1.901 & 5.23 & 24 & 5.015 & 5.86 \\
\hline 57 & 2.028 & 5.22 & 34 & 5.168 & 5.89 \\
\hline $2 \mathrm{I}$ & 2.162 & 5.23 & 43 & 5.364 & 5.95 \\
\hline 5 & 2.293 & 5.27 & 50 & $5 \cdot 5 \circ 3$ & 5.91 \\
\hline 27 & 2.425 & 5.28 & I 6 & 5.612 & 5.93 \\
\hline 38 & 2.619 & $5 \cdot 32$ & 60 & 5.676 & $5.9 \mathrm{I}$ \\
\hline 9 & 2.807 & 5.39 & 32 & 5.746 & 5.89 \\
\hline 22 & 2.998 & 5.45 & 47 & 5.793 & 5.88 \\
\hline & $3.21 \mathrm{I}$ & 5.43 & $I$ & $5.84 I$ & 6.01 \\
\hline
\end{tabular}

Nr. Fase Gr.

$55 \quad 5^{\mathrm{d}} \because 899 \quad 6^{\mathrm{m}}: 00$

$\begin{array}{lll}39 & 5.959 & 6.01\end{array}$

$\begin{array}{lll}45 & 6.052 & 6.08\end{array}$

$\begin{array}{lll}35 & 6.166 & 6.06\end{array}$

$\begin{array}{lll}29 & 6.304 & 5.97\end{array}$

$\begin{array}{lll}5 \mathrm{I} & 6.454 & 5.97\end{array}$

$2 \quad 6.634 \quad 5.93$

$\begin{array}{lll}17 & 6.776 & 5.86\end{array}$

$\begin{array}{llll}26 & 6.876 & 5.89\end{array}$

$\begin{array}{lll}58 & 6.98 \text { I } & 5.86\end{array}$

$\begin{array}{lll}12 & 7.083 & 5.80\end{array}$

$\begin{array}{lll}25 & 7.207 & 5.70\end{array}$

$\begin{array}{lll}18 & 7.378 & 5.68\end{array}$

$48 \quad 7.565 \quad 5.53$

$\begin{array}{lll}56 & 7.733 & 5.50\end{array}$

40 $7.896 \quad 5.43$

$30 \quad 8.100 \quad 5.37$

$448.16 \mathrm{I} \quad 5.3 \mathrm{I}$

$19 \quad 8.434 \quad 5.3 \mathrm{I}$

$\begin{array}{lll}3 & 8.628 & 5.30\end{array}$

G. B. Lacchini.

Aufforderung. Für eine Untersuchung über Fixsternparallaxen bitte ich um Zusendung der noch nicht oder an schwer zugänglichen Stellen veröffentlichten Parallaxenbestimmungen, soweit sie Parallaxen über o."o4 betreffen.

F. Haas, Universitäts-Sternwarte Berlin-Babelsberg in Neubabelsberg.

Anzeige. Zu kaufen gesucht Bonner Beobachtungen Bd. 3, 4, 5, 8. Angebote vermittelt die Redaktion.

Inhalt zu Nr. 5 12I. H. Rosenberg. Photometrische Messungen der Mondoberfläche und das Flächenphotometer der Sternwarte Österberg. 137. - H. Seeliger. Bemerkungen zum Aufsatz des Herrn van Rhijn in AN 5091. 145. - G. B. Lacchini. Osservazioni a stima della Variabile S Sagittae. 149. - F. Haas. Aufforderung. 15 I. - Anzeige. 15 I.

Mit Tafel I. Geschloesen 1921 Sept. 2x. Herauggeber: H. Kobolin. Druck von C. Schaidt. Fixpedition: Kiel, Moltkestr, 8o. Postscheck-Konto Nir. 6238 Hamburg Ix. 